\title{
STATISTICAL CHARACTERIZATION OF LEARNABLE SEQUENCES
}

P.H. Fuchs

Fachbereich Mathematik, Universität Frankfurt a.M.

Throughout this paper $x$ denotes the binary alphabet $\{0,1\}, x^{*}$ the set of (finite) words over $x, x^{\infty}$ the set of infinite binary sequences; $\Lambda$ denotes the empty word and $|x|$ denotes the length of $x \in X^{*}$.

First we present some informal considerations concerning learnability of infinite $0-1$-sequences. Let $R \subseteq X^{*} \times X^{*}$ be a rec. en. relation (an effective system of descriptions; sometimes called operator); we say $y$ describes $x$ iff $y R x$. We suppose that $R$ is an universal rec. en. relation, $i . e$. if $S$ is any rec. en. relation, there is $w \in X^{*}$ such that $\forall y, x: y S x \Rightarrow$ wyRx. Now, if $z \in . . m^{m}$ is a recursive sequence, there is a "best" way to describe $\mathrm{z}$ : there is $v \in \mathrm{X}^{*}$ such that $\forall \mathrm{n} \in \mathrm{N}$ : $\mathrm{vRz}^{\mathrm{n}}$ (where $z^{n}$ denotes the initial segment $z_{1} \ldots z_{n}$ of $z$ ). But usually (if $z$ is not recursive) the length of the description of $z^{n}$ will become larger as $n$ grows, because more and more additional information must be provided by the description. We call z learnable, if there is a "best" way to describe all the initial segments $z^{n}$ of $z$. The problem to characterize learnable sequences is due to R.P. Daley [1].

Let us pass to the formal definitions. Following Levin [3], we restrict the systems of descriptions to monotonic operators. A rec. en. relation $A \subseteq X^{*} \times X^{*}$ is called monotonic operator iff

(1) $\forall(y, x) \in A: \forall(v, u) \in A: \quad y \sqsubset v \rightarrow x[u \vee u[x$.

(We write $y\left[v\right.$ iff $v \in \mathrm{y}^{*}$ )

There is an universal monotonic operator $U$; that means: if $A$ is any monotonic operator, then $w \in X^{*}$ can effectively be found such that $\forall(y, x) \in A$ : $(w y, x) \in U$. We $f i x$ an universal monotonic operator $U$, a partial recursive function $\varphi: X^{*} \times X^{*} \rightarrow X$ such that $\operatorname{domain}(\varphi)=U$ and a running time function $\Phi$ for $\varphi$. For technical reasons we assume

(2) $\forall y, x: \Phi(y, x) \geq \log |y|$.

Notations:

(3) $\mathrm{Km}(\mathrm{x}):=\min \left\{|\mathrm{y}| \mid \mathrm{y} \in \mathrm{X}^{*} \wedge \mathrm{yUx}\right\}$; $\mathrm{Km}$ is called monotonic operator complexity. 
(4) Let $T$ be a growth function (i.e. $T$ is recursive, isotonic, unbounded).

$M_{x}^{T}:=\left\{y \in X^{*}|\quad \forall i s| x|: \exists k s| y \mid: y^{k} U x^{i} \wedge \Phi\left(y^{k}, x^{i}\right) \leq T(i)\right\}$.

Condition (2) ensures that there is a finite set $\widetilde{M}_{X}^{T} \leq X^{*}$ such that $M_{X}^{T}=$ $\tilde{M}_{X}^{T} x^{*}$ and $y \in \tilde{M}_{X}^{T} \Rightarrow \log |y| \leq T(|x|)$.

(5) $\mathrm{Km}^{\mathrm{T}}(\mathrm{x}):=\min \left\{|\mathrm{y}| \mid \mathrm{y} \in \mathrm{M}_{\mathrm{x}}^{\mathrm{T}}\right\}$.

$\mathrm{Km}^{\mathrm{T}}$ is recursive for sufficiently large functions $\mathrm{T}$.

In order to give a short definition of learnability we introduce a relation $s^{*}$ for (arbitrary) functions $f_{1}, f_{2}: N \rightarrow N$. We write $f_{1} s^{*} f_{2}$ iff $\forall g$ growth funct.: $\forall n: f_{1}(n) \leq f_{2}(n)+g(n)$.

\section{Definition:}

$z \in X^{\infty}$ is called learnable iff there is a growth function $T$ such that the function $\mathrm{n} \rightarrow \mathrm{Km}^{\mathrm{T}}\left(\mathrm{z}^{\mathrm{n}}\right)$ is $s^{*}$-minimal among $\left(\mathrm{n} \rightarrow \mathrm{Km}^{t}\left(\mathrm{z}^{\mathrm{n}}\right) \mid \mathrm{t}\right.$ growth funct.).

An equivalent, but perhaps more intuitive definition of learnability makes use of the notion of a recursive coding. A recursive function $\psi: x^{*}+x^{*}$ is called a recursive coding, iff $\forall x \in X^{*}: \psi(x)$ Ux. Thus $\psi(x)$ describes $x$; and $z \in x^{\infty}$ is learnable iff there is a recursive coding $\psi$ such that $n+\left|\psi\left(z^{n}\right)\right|$ is $s^{*}$-minimal among $\left(n \rightarrow\left|\psi^{\prime}\left(z^{n}\right)\right| \mid \psi^{\prime}\right.$ rec. coding).

There is a rec. en. sequence $z$ which is not learnable [2], [4]. Moreover this sequence $z$ can be chosen such that it satisfies the following 1.0 . cut-down property.

For every growth function $T$ there exists a growth function $T^{\prime}$ such that $\forall q<1: \exists^{\infty} n: \mathrm{Km}^{\mathrm{T}^{\prime}}\left(\mathrm{z}^{\mathrm{n}}\right) \leq \mathrm{Km}^{\mathrm{T}}\left(\mathrm{z}^{\mathrm{n}}\right)-\mathrm{q} \cdot \mathrm{n}$.

The attempt to characterize learnable sequences statistically arises from the following idea. Consider random sequences with respect to the equiprobability aistribution $\bar{j}$. These sequences are completely irregular; we suppose that the best way to describe their initial segments is to describe them by themselves. Recursive sequences are learnable but not at all $\mu$-random. Therefore we first generalize the concept of randomness in order to cover both cases.

Let $\left.p: x^{*} \rightarrow\right] 0,1[\cap Q$ be a recursive function. $p$ defines a recursive probability measure on $x^{\infty}$ (i.e. on the $\sigma$-algebra generated by the sets $\mathrm{xx}^{\infty}$ ) as follows.

$\bar{p}\left(x x^{\infty}\right):=\prod_{x_{i+1}=1} p\left(x^{i}\right) \cdot x_{i+1}=0\left(1-p\left(x^{i}\right)\right)$. 
We call p a recursive probability measure (r.p.m.), too. $\mu$ denotes the equiprobability distribution $\forall x \in x^{*}: \mu(x)=2^{-1}$.

A recursive p-martingale is a recursive function $V: X^{*} \rightarrow Q_{+}$satisfying $\forall x \in X^{*}: V(x)=p(x) V(x 1)+(1-p(x)) V(x o)$. In analogy to the $\mu$-case [5] we define p-randomness.

\section{Definition:}

Let $p$ be a r.p.m.. $z \in x^{\infty}$ is not p-random iff $\exists V$ rec. p-martingale: $\exists h$ growth function: $\exists^{\infty} n: V\left(z^{n}\right) \geq h(n)$.

We call $\mathcal{K}:=\left\{z \in \mathrm{X}^{\infty} \mid \exists \mathrm{p}\right.$ r.p.m.: $\mathrm{z}$ is $\mathrm{p}$-random $\}$ the set of general random secuences.

Let $z$ be a recursive sequence. We define

$$
p\left(z^{n-1}\right)=\left\{\begin{array}{l}
\left(2^{n}+1\right) \cdot\left(2^{n}+2\right)^{-1}, \text { if } z_{n}=1 \\
\left(2^{n}+2\right)^{-1}, \text { if } z_{n}=0
\end{array}\right.
$$

It is easily seen that $z$ is p-random. Thus, every recursive sequence is a general random sequence. Martingales and monotonic operator complexity are strongly related as the following theorem shows.

\section{Theorem 1.}

(a) Let $T$ be a growth function. There is a recursive $\mu$-martingale $V>0$ such that $\forall x \in X^{*}: \log V(x) \geq|x|-k^{T}(x)$.

(b) Let $V$ be a recursive $\mu$-martingale, $V>0$. There is a growth function $T$ and a constant $c$ such that $\forall x \in X^{*}: \log V(x) \leq|x|-\mathrm{Km}^{\mathrm{T}}(\mathrm{x})+\mathrm{c}$.

\section{Eroof:}

(a) Define $\bar{V}(x):=\bar{\mu}\left(M_{x}^{T} x^{\infty}\right) \cdot 2|x|$. From condition (2) we conclude that $\bar{V}$ is recursive. $\bar{V}$ is a recursive $\mu$-submartingale, i.e. $\forall x \in X^{*}: \bar{V}(x) \geq$ $2^{-1} \cdot\left(\bar{V}\left(x_{0}\right)+\bar{V}\left(x_{1}\right)\right)$. Therefore we can construct a recursive $\mu$-martingale $\mathrm{V} \geq \bar{V}$. V satisfies $(\mathrm{a})$ : from $\mathrm{y} \in \mathrm{M}_{\mathrm{x}}^{\mathrm{T}},|\mathrm{y}|=\mathrm{Km}^{\mathrm{T}}(\mathrm{x})$ we conclude $\bar{\mu}\left(\mathrm{M}_{\mathrm{x}}^{\mathrm{T}} \mathrm{x}^{\infty}\right) \geq$ $2^{-|y|}$ and this implies $\log V(x) \geq|x|-\mathrm{Km}^{\mathrm{T}}(\mathrm{x})$.

(b) We define a r.p.m. $p$ as follows: $p(x):=V(x 1) / 2 \cdot V(x)$. It suffices to find a growth function $T$ such that $\mathrm{Km}^{\mathrm{T}}(\mathrm{x}) \leq|\log \overline{\mathrm{p}}(\mathrm{x})|+c$, for we have $V(x)=2|x| \bar{p}(x)(V(\Lambda))^{-1}$.

First we define a mapping $F: X^{*} \rightarrow X^{*}$ using the following notations. For $i=1,2,3, \ldots$ we divide the interval $[0,1]$ by $2^{i}-1$ equidistant points into $2^{i}$ open intervals of length $2^{-1}$; these intervals are denoted by $I_{k}^{i}$ $\left(k=1, \ldots, 2^{i}\right)$. To every interval of this kind we assoziate a finite binary sequence by the function $B$ in the following way: 
$B\left(I_{k}^{i}\right)=$ the (lexicographically) $k$-th sequence in $x^{i}$. Fact $1: I_{k}^{i} \geq I_{r}^{i} \Leftrightarrow B\left(I_{k}^{i}\right)\left[B\left(I_{r}^{i}\right)\right.$.

To define $F$ at $x \in X^{*}$ we consider the interval $\left.I_{X}=\right] \sum_{y<x} \vec{p}(y), \sum_{y \leq x} \bar{p}(y)[$, $y \in x^{n} \quad y \in x^{n}$

where $x<y$ denotes the lexicographical ordering and $\bar{p}(y):=\bar{p}\left(y x^{\infty}\right)$. Let $I_{k}^{i}$ be an interval of maximal length contained in $I_{x}$, i.e. $I_{k}^{i} \subseteq_{X} I_{X}$ $\forall j<i: \forall r\left(0<r \leq 2^{j}\right): I_{r}^{j} \notin I_{x}$. There is one and only one such $I_{k}^{i}$. Then we set $F(x):=B\left(I_{k}^{\frac{1}{k}}\right)$.

Fact 2: $\forall x:|F(x)| \leq\lceil-\log \bar{p}(x)\rceil+1$. The length of $I_{x}$ is $\bar{p}(x)$; therefore $i \leq\lceil-\log \bar{p}(x)\rceil+1$.

Fact $3: F: X^{*} \rightarrow X^{*}$ is recursive.

Fact $4: F^{-1}$ is a monotonic operator.

Let $F(x)\left[F\left(x^{\prime}\right)\right.$. Applying fact 1 we conclude for the intervals $B^{-1}(F(x))$ and $B^{-1}\left(F\left(x^{\prime}\right)\right): B^{-1}(F(x)) \geq B^{-1}\left(F\left(x^{\prime}\right)\right)$. Then $x^{\prime} \sqsupset x$ must hold, for $x^{\prime} \not x \wedge x \not x^{\prime}$ would imply $I_{x^{\prime}} \cap I_{x}=\varnothing$.

Choose $v \in X^{*}$ such that $\forall x, y \in X^{*}: y=F(x) \Rightarrow v y U x$. Define $T(n):=$ $\max \left\{(\mathrm{vF}(\mathrm{x}), \mathrm{x}) \mid \mathrm{x} \in \mathrm{X}^{\mathrm{n}}\right\}$. Fact 3 implies that $\mathrm{T}$ is recursive. From fact 2 we conclude $\left.\forall \mathrm{x} \in \mathrm{X}^{*}: \mathrm{Km}^{\mathrm{T}}(\mathrm{x}) \leq|\mathrm{F}(\mathrm{x})|+|\mathrm{v}| \leq \mid-\log \overline{\mathrm{p}}(\mathrm{x})\right\rceil+1+|\mathrm{v}|$.

Theorem 1 is a counterpart. of Levin's theorem 2 [3]; in [4] theorem 1 is used to characterize p-randomness in terms of $\mathrm{km}$.

Theorem 2. (Statistical characterization of Learnable sequences)

For every $\mathrm{z} \in \mathrm{X}^{\infty}$ the following conditions are equivalent.

(a) $z$ is learnable

(b) $z$ is a general random sequence.

\section{Eroof:}

$"(a) \Rightarrow(b) "$ : Let $T$ be a growth function which learns $z$. Apply thm.1(a) to $T$. We find a recursive $\mu$-martingale $V>0$ s.th. $\log V(x) \geq|x|-\mathrm{Km}^{\mathrm{T}}(\mathrm{x})$. Define $p(x):=V(x 1) / 2 \cdot V(x), p$ is a r.p.m.. We claim that $z$ is $p$-random.

Assume $z$ is not p-random. Then there is a recursive p-martingale $\bar{V}$ and a growth function $g$ s.th. $\exists^{\infty} n: \bar{V}\left(z^{n}\right) \geq g(n)$. We define a recursive $u-$ martingale $V^{\prime}(x):=\bar{V}(x) \cdot \bar{p}(x) \cdot 2|x|$. From thm. $1(b)$ we get a growth function $T^{\prime}$ such that $\log V^{\prime}(x) \leq|x|-K^{T^{\prime}}(x)+c$. Using the identity $\bar{p}(x)=$ $(V(M))^{-1} \cdot V(x) \cdot 2^{-|x|}$ we obtain $\log \bar{V}(x)+\mathrm{Km}^{\prime}(x) \leq \mathrm{Km}^{\mathrm{T}}(x)+c+\log V(\Lambda)$; and this implies $\exists^{\infty} n$ : log $g(n)-c^{\prime}+K^{T}{ }^{\prime}\left(z^{n}\right) \leq \mathrm{Km}^{T}\left(z^{n}\right)$, which is contradictory to the supposition that $T$ learns $z$. 
$"(b) \Rightarrow(a) "$ : Let $z$ be p-random. Consider the $\mu$-martingale $V(x)=2|x| \bar{p}(x)$ and apply thm, $1(b)$. We obtain $T$ and $c$ such that $o \leq|\log \bar{p}(x)|-\operatorname{Km}^{T}(x)+c$. We show that $T$ learns $z$. If not, there would be $T$, and a growth function $g$ such that $\exists^{\infty} \mathrm{n}$ : $\mathrm{Km}^{\mathrm{T}^{\prime}}\left(\mathrm{z}^{\mathrm{n}}\right)+\mathrm{g}(\mathrm{n}) \leq \mathrm{Km}^{\mathrm{T}}\left(\mathrm{z}^{\mathrm{n}}\right)$. Applying thm.1(a) to $\mathrm{T}^{\mathrm{t}}$ we obtain a recursive $\mu$-martingale $V^{\prime}$ such that $\exists^{\infty} n: g(n) \leq \log V^{\prime}\left(z^{n}\right)+$ $\left|\log \bar{p}\left(z^{n}\right)\right|-n+c$. But this is contadictory to the p-randomness of $z$, for $\bar{V}(x):=2^{-|x|} \cdot V^{\prime}(x) \cdot(\bar{p}(x))^{-1}$ is a recursive p-martingale.

A learnable sequence $z$ may have many algorithmically recognizable regularities; but if $T$ learns $z$, then all these regularities are found within running time $T$. Beyond that, $z$ must be quite irregular. The characterization theorem shows that all regularities of a learnable sequence can be completely condensed into a r.p.m. p.

The correspondence between growth functions and r.p.m.'s is effective in the sense that given $T$ (which learns $z$ ), one can effectively find a r.p.m. p such that $z$ is p-random and vice versa.

The set of non-learnable sequences, i.e. $x^{\infty} \cdot \nVdash$ is rather small. In fact, we have $\forall \mathrm{p} r . \mathrm{p} . \mathrm{m} .: \overline{\mathrm{p}}\left(\mathrm{x}^{\infty}, x\right)=0$.

References:

[1] R.P. Daley

THE PROCESS COMPLEXITY AND THE UNDERSTANDING OF SEQUENCES. Proceedings of Symposium and summer School MFCS, High Tatras, September 3-8, 1973.

[2] P.H. Fuchs PROGRAMMKOMPLEXITÄT, ZUF̈̈LLIGKEIT , LERNBARKEIT. Dissertation, Frankfurt 1975.

[3] I.A. Levin

[4] C.P. Schnorr ON THE NOTION OF A RANDOM SEQUENCE. Soviet Math. Dokl. 14, 1973.

P.H. Fuchs THE COINCIDENCE OF IEARNABLE, OF OPTIMALLY COMPRESSIBLE AND OF GENERAL RANDOM SEQUENCES. Preprint, Fachbereich Mathematik, Universität Frankfurt 1975.

[5] C.P. Schnorr ZUFALIIGKEIT UND WAHRSCHEINLICHKEIT. Lecture Notes in Math., vol 218, Berlin and New York, 1971. 